\title{
Digital image correlation techniques for measuring tyre-road interface parameters: Part 2 - Longitudinal tyre slip ratio measurement
}

Theunis R. Botha* \& P. Schalk Els

Department of Mechanical and Aeronautical Engineering, University of Pretoria, co Lynnwood Road and Roper Street, Pretoria, 0002, South Africa

\author{
* Corresponding author. \\ E-mail address: trbotha@tuks.co.za \\ Telephone: +27124203289 \\ E-mail address: \\ trbotha@tuks.co.za (Theunis R. Botha) \\ schalk.els@up.ac.za (P. Schalk Els)
}

\begin{abstract}
Measurement of tyre longitudinal slip-ratio is often estimated from three independent measurements devices namely wheel rotation speed, vehicle speed and tyre rolling radius. This produces an expensive measurement system to indirectly determine the slip-ratio. This paper presents a method by which the slip-ratio is determined from a video camera using digital image correlation techniques. The camera, mounted in such a way that the contact patch region is captured, enables the system to measure the tyre tread speed and ground speed at the contact patch. The slip-ratio is then determined from these two measurements.
\end{abstract}

\section{INTRODUCTION}

The mechanism for generating tyre longitudinal forces is an exceedingly complex process which occurs as a result of the relative sliding motion between a pneumatic tyre and the road. While the sliding motion is nonuniform across the contact patch [1], with regions where the contact patch adheres to the surface and regions where it slides, it can be shown that the generated longitudinal force can be sufficiently described as the ratio of the average relative speed between the tyre and the ground and the vehicle speed. This parameter is termed the wheel slip-ratio [2]. This slip-ratio is essential when constructing tyre longitudinal models and can be a useful 
measurement in vehicle stability programs such as traction control, ABS, ESP, and other. The slip ratio definition often used in many studies is given as [3]:

$$
S=-\frac{\left(V_{t}-V_{x}\right)}{V_{t}}
$$

where $S$ is the slip ratio as a fraction, $V_{t}$ is the theoretical (at zero slip) speed at the center of the wheel, $V_{x}$ is the actual tyre speed. The theoretical tyre speed $V_{t}$ is often merely represented as the vehicle longitudinal speed $V$. The actual tyre speed is determined as:

$$
V_{x}=\omega R_{e}
$$

where $\omega$ is the tyre angular velocity and $R_{e}$ is the effective tyre radius.

In experimental laboratory tests aimed at charactering longitudinal tyre models, both the tyre and the surface are controlled accurately to move at known speeds, thus by measuring the tyre radius the wheel slip-ratio can be accurately determined. The contact surface of these test setups typically consists of a rotating drum [4]. Drum tests however have the disadvantages that the surface is usually smooth and not representative of asphalt and the tyre makes contact with a circular surface as opposed to a flat surface. While a flat track testing machine [5], alleviates the problem of a rounded surface, the surface however usually still consists of a steel belt sometimes coated with an artificial surface. Additionally these testing machines are exceedingly expensive and are quite difficult to scale for larger tyres used in mining and agriculture.

An alternative to laboratory testing is in-situ testing, on real road surfaces, conducted using a tyre test machine or the actual vehicle the tyres are used on. This methodology alleviates the problems associated with laboratory testing. The most difficult task however is the accurate estimation of the wheel slip-ratio.

Traditionally the wheel slip-ratio is computed from (1) by measuring the three parameters separately using three measurement devices. While it is fairly inexpensive to accurately measure the wheel rotational speed, the rotation speed is measured at the rim and not at the contact patch. Additional filtering also needs to be applied to the signal. Accurate measurements of the vehicle speed and effective tyre roll radius are more expensive to obtain. Miller et al [6] shows how the slip-ratio can be determined using GPS. To obtain accurate GPS measurements at a high sampling frequency $(50-100 \mathrm{~Hz})$ however requires an expensive differential GPS receiver. It should also be noted that GPS signals can have a latency of up to $150 \mathrm{~ms}$ effecting real time results. The tyre rolling radius is most often not measured but rather assumed constant. While, Miller et al [6], determine the rolling radius using the GPS vehicle speed and wheel speed, this can only be accomplished under 
no-slip i.e. when no braking is performed. Thus, for the entirety of the braking tests the rolling radius is assumed constant, however load transferred due to braking has an effect on the tyre rolling radius. It is therefore important to continuously measure the rolling radius.

Since wheel speed is measured at the rim, and not the contact patch, the estimated slip-ratio is assumed to be produced immediately at the contact patch. The generated longitudinal force however has a small phase lag compared to the slip-ratio, [7], [8]. The phase lag is partially attributed to the transient transfer of the longitudinal forces generated at the contact to the wheel hub due to the tyre inertia. The transient formation of the forces at the contact patch is often neglected. The cause of this transient buildup of forces at the contact patch is not well understood, and is partially associated with the viscoelastic nature of the thread and the generation of the slip condition at the contact patch [9], pertaining to the deformation of the tread and side wall [10].

In this paper a method whereby the tyre slip-ratio is measured at the contact patch is presented. The method comprises of a single video camera, mounted in such a way to a tyre test machine or vehicle so that it captures both the tyre and the road at the tyre-road interface. The slip-ratio is measured by performing digital image correlation techniques on consecutive frames of the contact patch encompassing both the tyre and road.

\section{DIGITAL IMAGE CORRELATION TECHNIQUE}

Digital Image Correlation (DIC) is a method whereby optical methods are used to track changes in an image. These methods can be used to obtain a variety of measurements ranging from displacements and velocities of particles to the strain of an object in an image [11]. There are numerous methods which can be used to perform DIC, however the underlying methodology remains the same. The techniques try to match regions in one image to regions in another effectively tracking these regions across images. Often these images make up a time sequence of images, $I_{t}[\mathrm{t}=0,1, \ldots, \mathrm{n}]$, taken with a constant time difference apart. In strain based measurements the displacement field is computed across the whole image, however to simplify and speed up the DIC smaller key point regions can be tracked as opposed to the complete image.

To track a subset of regions from the image, first requires the identification of unique key points in both images. These key points are locations which should be unique i.e. easily differentiable from other key points, and would therefore be easy to identify in other images. In (reference Part 1) a simplistic feature and tracking 
algorithm is used based on tracking corner features. This algorithm however failed to consistently track a sufficient amount of features needed to perform the analysis. This is due to the assumptions made by the algorithm being violated in this particular case. As a result a more complex feature descriptor technique is used. Feature descriptors use a different means to obtain unique key points. From these key points features which uniquely identify these regions are then extracted, effectively providing each key point with a fingerprint. The method of obtaining key points and the type of features extracted often makes up the main difference between DIC algorithms. Two robust feature descriptors often used are Scale-Invariant Feature Transform (SIFT) [12] and Speeded Up Robust Features (SURF) [13].

In this paper the SIFT feature descriptor is used. SIFT key points are determined by local minima and maxima from the result of applying difference of Gaussians to the image. This is performed on numerous scaled and blurred images from the same scene to obtain regions with high contrast that are scale invariant. An orientation is also assigned to each key point based on the direction of the local image gradient to obtain invariance to rotation. After key points have been assigned features are extracted around this region. The features are the magnitude and direction of the image gradient in a window of $4 \times 4$, where each grid point describes the magnitude of the gradient in 8 directions. In total 128 features are extracted per key point which creates a feature vector. The feature vector is normalized to obtain invariance to illumination.

An attempt is then made to match the key points from the two separate images therefore tracking the key points from one image to another. This is performed by using a suitable distance metric between features, usually the Euclidean metric. The match of a key point in image $I_{t}$ is then obtained by determining the distances between key points in image $I_{t+1}$ either using an exhaustive brute force method or a random sampling method. The key point with the smallest distance is taken as the matching key point. If the key points are extracted from two consecutive timed images the velocity in terms of pixels/ second can be obtained for the key points.

\section{SLIP-RATIO FROM DIGITAL IMAGE CORRELATION}

The slip ratio is determined by post processing a sequence of images obtained from a single camera. The camera is initially calibrated to remove any distortion caused by the lens. Particles on both the tyre and the road, close to the contact patch, are tracked using the DIC method described above. It is important that the mounting of the camera is low enough such that the bottom of the tyre in contact with the road is visible. To prevent damage to the camera it can be place on the inside of the vehicle close to the suspension which would prevent 
obstacles from hitting the camera. The orientation is not so important as the parallax error is corrected for in the algorithm discussed below. A small window is selected in image $I_{t}$ which encapsulates both the tyre and road close to the contact interface. First key points are identified inside this small window. These key points are then tracked by identifying key points all over image $I_{t+1}$. The key points from images $I_{t}$ and $I_{t+1}$ are matched to obtain the pixel speed of these key points. Figure 1 shows the tracking of the key points from image $I_{t}$ on the left to image $I_{t+1}$ on the right, with matched key points joined with a line across the two images.

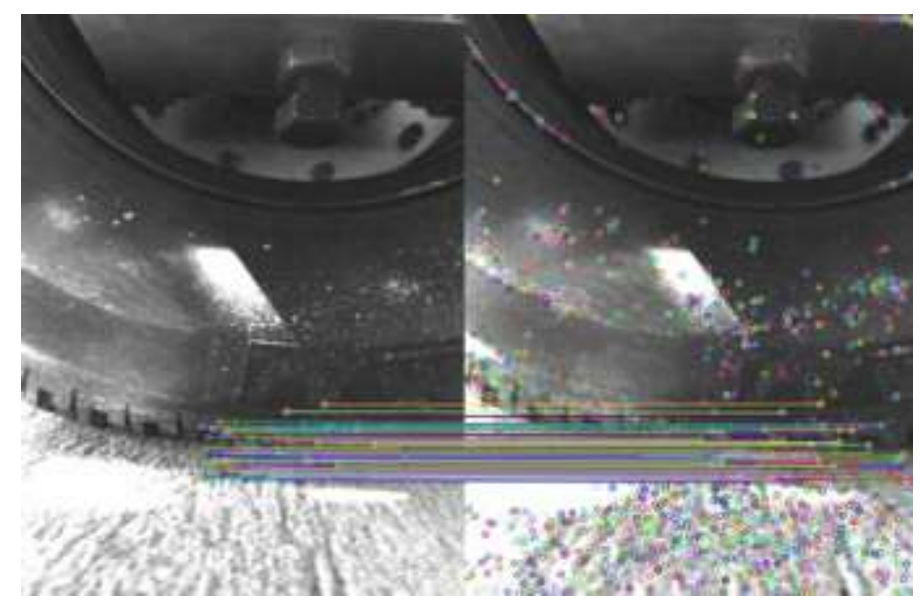

Figure 1: Tracking of key points from contact patch in image $\boldsymbol{I}_{\boldsymbol{t}}$ (left), to image $\boldsymbol{I}_{\boldsymbol{t}+\mathbf{1}}$ (right), and matched key points are joined with a line across the two images.

The DIC method used point can at times produce mismatched key points. In order to reduce the mismatched key points, an initial outlier rejection is performed. Since the speed of the regions under consideration should be mostly horizontal, matches that result in large deviation in the y-direction are rejected, i.e. key points which move too much in the vertical direction.

Once the key points have been matched they have to be separated into three categories, points lying on the tyre, points lying on the road and any additional outliers. At first a simplistic method was proposed whereby a horizontal line is drawn at the contact point, any points above this line is on the tyre and any points below are on the road. This method, while being very efficient and successful at the start of the test, causes some points to be categorized incorrectly due to the contact point moving vertically during the test. The method also fails to remove any additional outliers which are present. The vertical movement of the contact patch is due to the roll radius changing as a result of load being transferred during braking as well as the test rig bouncing. A more robust method for categorizing the pixels is therefore required. 
In this paper a clustering technique is implemented to characterize the key points into the three categories. Two clustering methods generally used are the K-means and agglomerative clustering technique [14]. In the Kmeans approach $\mathrm{K}$ cluster centroids are chosen and all points are joined to these centroids, the centroids are updated for each cluster, after which all points are joined to the new centroids. This process continues until the centroids change very little. The disadvantage of this is that the process continues until steady state is reached. In the agglomerative clustering technique each point is considered as a cluster, iteratively the two clusters which are the closest are joined and the cluster centroid is recalculated. The process is repeated until the desired numbers of clusters are obtained. The disadvantage of this method is that prior knowledge of the number of clusters is required. In this application while we require three groups, the situation could arise when there is no outlier cluster, resulting in two clusters containing either tyre or road points. An alternate case is where there may be more than one outlier cluster, with one cluster closer in distance to either the tyre cluster or the road cluster rather than another outlier cluster. This would result in outliers being clustered together with either the tyre or road clusters.

The clustering algorithm implemented in this paper includes aspects from both clustering techniques. Two main clusters are initially created, a tyre and a road cluster, containing initial points in each cluster. The remaining key points are considered to be an individual cluster themselves. The distance from each cluster to one of the two main clusters is calculated. The cluster with the smallest distance to one of the main clusters is then included into the corresponding main cluster. The cluster centroid is recalculated along with the distance to each of the remaining individual clusters. This process is repeated until the smallest distance between exceeds some threshold. The remaining clusters, not included into one of the main clusters, are considered outliers. This process is graphically illustrated in Figure 2. The initialisation creates the initial tyre (cluster 1) and road (cluster

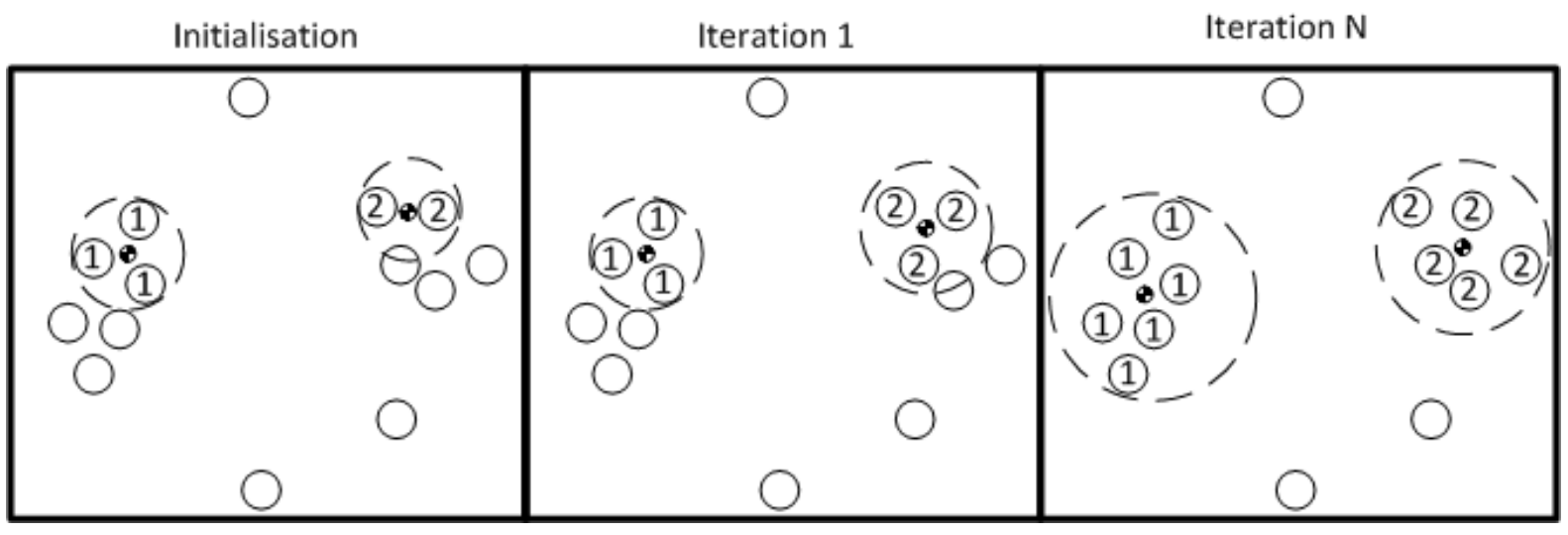

Figure 2: Clustering algorithm. Initial tyre and road clusters are created (left). Points are then iteratively merged into these clusters based on distance to cluster (middle). Process continues until the distance to a cluster exceeds some threshold (right). 
2) clusters. Iteratively the point closest to any of these two clusters is joined into that cluster. In the first iteration a point is joined into cluster 2. The centre of this cluster is recalculated and the distance to each point is recalculated. This process is continued for $\mathrm{N}$ iterations until the distance of a point to a cluster is larger than a threshold. The final clusters are then obtained with the remaining points classified as outliers.

The distance metric used is the Euclidean metric given as:

$$
p_{i j}=\sqrt{\sum_{k=1}^{d}\left(X_{i k}-X_{j k}\right)^{2}}
$$

where $p_{i j}$ is the Euclidean distance between clusters $i$ and $j$ in $d$ dimensional space. $X_{i k}$ is the $\mathrm{k}^{\text {th }}$ component of cluster $i^{\prime} s$ centroid. In this paper a three dimensional space is used to describe each cluster. The first component is the speed of the cluster, this component is used since the tyre and road should have different speeds, especially during slip. The second component is the x-pixel coordinate, from the image, where the cluster is associated. This component is included because the tyre and road are situated on different vertical locations on the image. The last component is the homography error of the cluster. Since there is a possible difference in magnitude in the three components, the components are normalized such that each component vector has a mean of zero and a standard deviation of one. The cluster center is simply taken as the mean of the cluster data points. The following section discusses homography and why it is included as a component of the clusters.

A homography is a one-to one mapping of a planar surface from one image to another. The homography is represented by a $3 \times 3$ matrix $\boldsymbol{H}$, with the mapping of image correspondences on a plane mapped as:

$$
H \boldsymbol{q}=\boldsymbol{q}^{\prime}
$$

Where $\boldsymbol{q}$ is the homogeneous coordinates of a correspondence, obtained as the pixel coordinates augmented by an additional 1 i.e. $\boldsymbol{q}=\left[\begin{array}{lll}x & y & z\end{array}\right]=\left[\begin{array}{lll}x_{p} & y_{p} & 1\end{array}\right]^{T}$ in image $I_{t}$, and $\boldsymbol{q}^{\prime}$ is the associated correspondence in image $I_{t+1}$. Although the homographic matrix contains 9 elements it is ambiguous up to scale and has only 8 degrees of freedom. Thus only 8 independent elements need to be solved for. The scale can be arbitrarily chosen or set such that $\|\boldsymbol{H}\|=1$. The homographic matrix is estimated from the fact that the cross product of $\boldsymbol{H q}$ and $\boldsymbol{q}^{\prime}$ is zero: 


$$
\boldsymbol{q}^{\prime} \times H \boldsymbol{q}=\left[\begin{array}{c}
y^{\prime} h_{3} q-z^{\prime} h_{2} q \\
z^{\prime} h_{1} q-x^{\prime} h_{3} q \\
x^{\prime} h_{2} q-y^{\prime} h_{1} q
\end{array}\right]=0
$$

Where $\boldsymbol{h}_{\mathbf{1}}$ is the first row of the homohraphy matrix $\boldsymbol{H}$. The third equation is a linear combination of the first two, therefore each correspondence provides two linearly independent constraints on the elements of $\boldsymbol{H}$. Thus, in order to estimate the homographic matrix $\boldsymbol{H}$ at least four correspondences are required which lie in a plane. Equation (5) can be rewritten as:

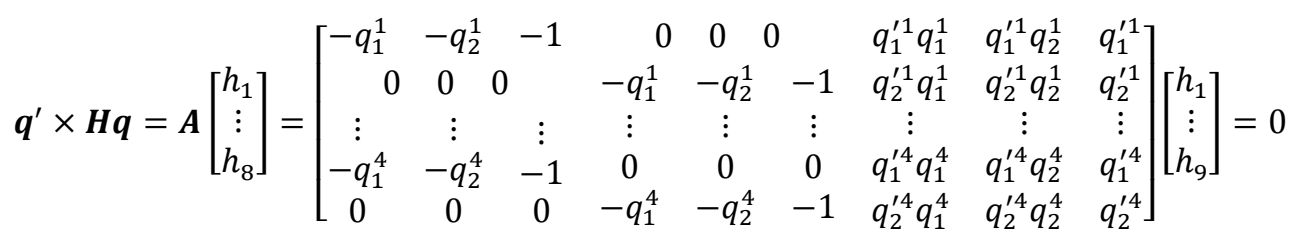

where $q_{i}^{j}$ is the $\mathrm{i}^{\text {th }}$ value of the $\mathrm{j}^{\text {th }}$ correspondence and $h_{k}$ is the $\mathrm{k}^{\text {th }}$ value of the homographic matrix. The homograhic vector in (6) is solved using Singular Value Decomposition (SVD) and the solutions is obtained from the right singular vector corresponding to the smallest singular value. An overview of this method is provided in [15].

The estimation is achieved by means of the RANdom SAmple Consesus (RANSAC) algorithm [16]. The RANSAC algorithm is an iterative procedure to estimate the parameters of a model from a set of data which includes outliers. The synopsis of the method is to randomly sample a minimum set of data points for which to develop the model, in this case four correspondences. The model is derived from the minimal set and each remaining data point is tested against the model using a suitable metric, in this case $p=\left\|\boldsymbol{q}^{\prime}-\boldsymbol{H q}\right\|$. If the error of the metric is within some suitable error the data point is considered to agree with the model and counted as an inlier. The process of sampling a minimum set of data points and developing a model continues for a suitable amount of iterations. Upon completion the model with the most inliers is returned as the correct model.

The homography is important as it can be used to model the road surface the tyre is rolling over under the assumption that the road can be represented as a plane. A key point which strongly agrees with the model is considered to be on the road and a point which does not is considered to lie off the plane or a mismatched outlier. To generate the homography, correspondences which are guaranteed to be part of the road plane are chosen. This is achieved be performing DIC on a region below the contact patch which only contains the surface the tyre is rolling over. The homography is also used to seed the road cluster with initial points. Key points with a homographic error below a certain threshold are considered to be on the road and included into this cluster, 
with the threshold being set very low to ensure only road point are selected. This process is repeated at each image $\boldsymbol{I}_{\boldsymbol{t}}$ to seed the initial road cluster with points.

The initial points on the tyre are selected from the first image $I_{0}$ based on their vertical pixel height being within a certain range above the initial road points, as well as their velocity magnitudes having similar values to the road points thus assuming an initial no slip condition. This process is only performed on the first image after which the cluster is seeded with points from the previous image $I_{t-1}$. The points from the previous image are selected to be the points with the highest vertical pixel coordinate, this is done to prevent the tyre cluster from migrating towards the road cluster. The points selected are also not allowed to be chosen to seed the tyre cluster in the next iteration. If no new points have been included into the cluster, then in the following iteration the tyre cluster is initialized using the same method as initializing the cluster using the first image $I_{0}$. The tyre -road contact point's vertical pixel coordinate is also determined at each iteration. This is accomplished by taking the distance halfway between the tyre and road clusters vertical pixel coordinate centres. However, instead of completely replacing the previous iterations estimate of the contact point height with the new estimate, the estimate is updated by a fraction of the error between new and previous estimate as:

$$
R_{y, t+1}=R_{y, t}+c\left(\bar{R}_{y, t+1}-R_{y, t}\right),
$$

where $R_{y, t+1}$ is the updated position of the contact point in the y-direction, $\bar{R}_{y, t+1}$ is the new estimate of contact point and $c$ is a constant describing the rate of update, $c \leq 1$. The tyre -road contact point is used to position the window from which the key points are generated to allow an equal amount of tyre and road region being included. This parameter also effectively means that the change in effective tyre roll radius is captured from the camera.

Figure 3 depicts one iteration showing images $I_{t}$ and $I_{t+1}$ on the left and right respectively. The left image shows the trace of each key point depicting the velocities of the key point as well as the windows from which key points are generated. The image on the right depicts the clustering result with the red points classified as tyre points, the blue points on the road and green points classified as outliers. The red horizontal line also depicts the tyre -road contact height. The figure shows excellent classification of both tyre and road points with no outliers contained within these groups. There are however some points which should be classified as either being a tyre or road points that are classified as outliers. This is of no concern as enough points are classified correctly with no inclusion of outliers. This method was found to be very robust at classifying the key points correctly while removing all outliers. 


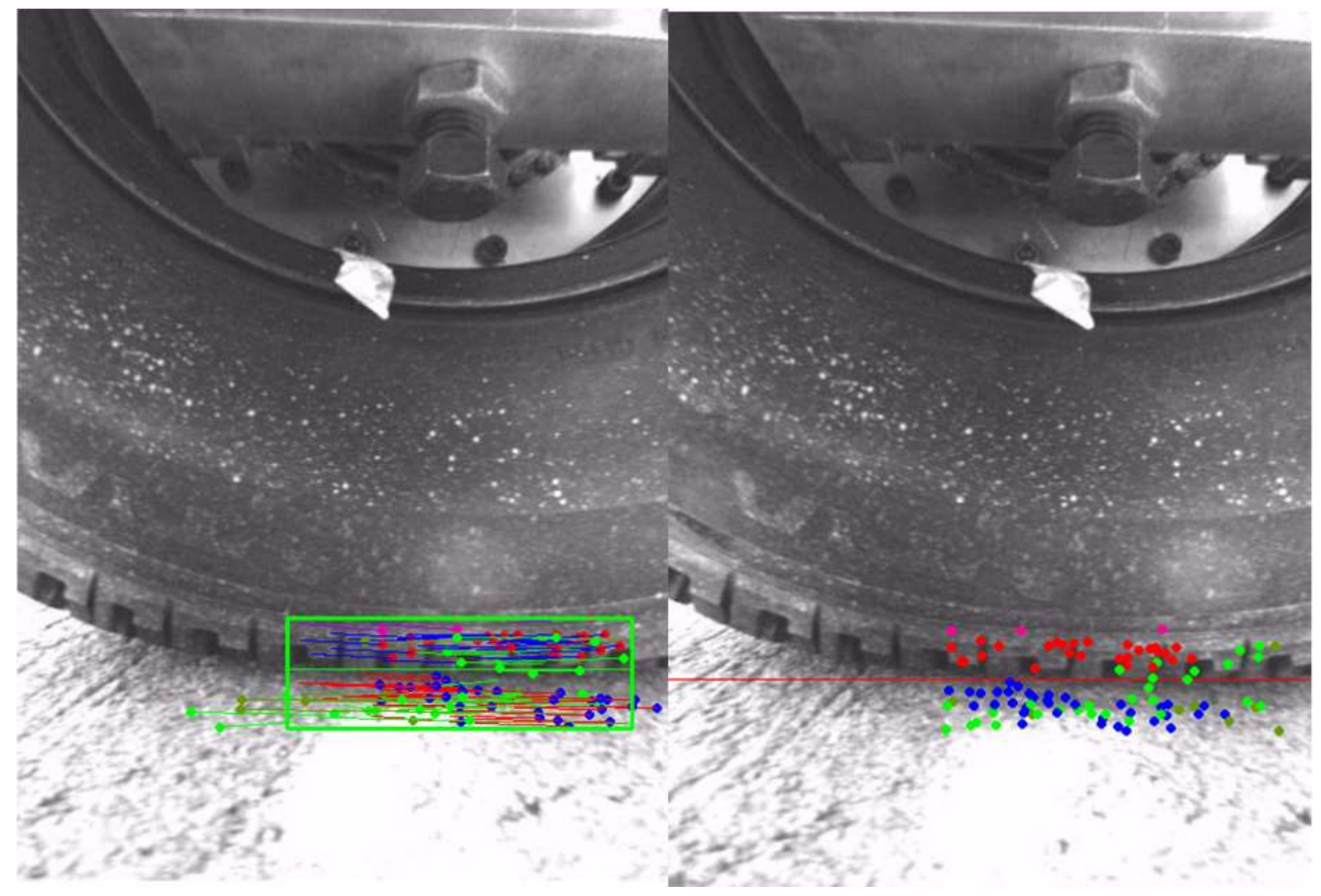

Figure 3: Tracking and classification of key points from contact patch in image $\boldsymbol{I}_{\boldsymbol{t}}$ (left), to image $\boldsymbol{I}_{\boldsymbol{t}+\mathbf{1}}$ (right). Points classified as tyre points have red dots and those on the road have blue dots. Outliers are coloured green.

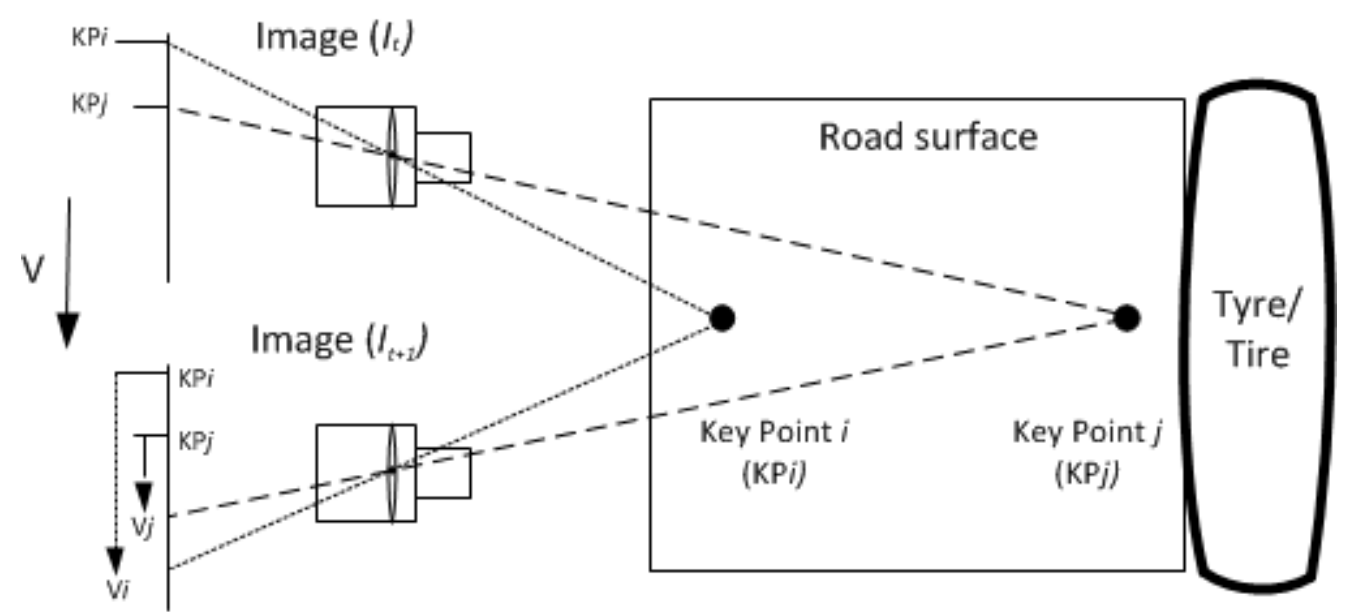

Figure 4: Illustration of the parallax effect caused by points having different depths from the camera.

After classification, the data points inside the clusters are used to obtain the velocity of the road and of the tyre at the contact point. One aspect which needs to be taken into consideration is the parallax effect caused by the difference in distance from the camera (depth) to the key points. This effect is illustrated in Figure 4, where key point $K P_{i}$ is closer to the camera than key point $K P_{j}$. As illustrated this causes the velocity measured at $K P_{i}$ 

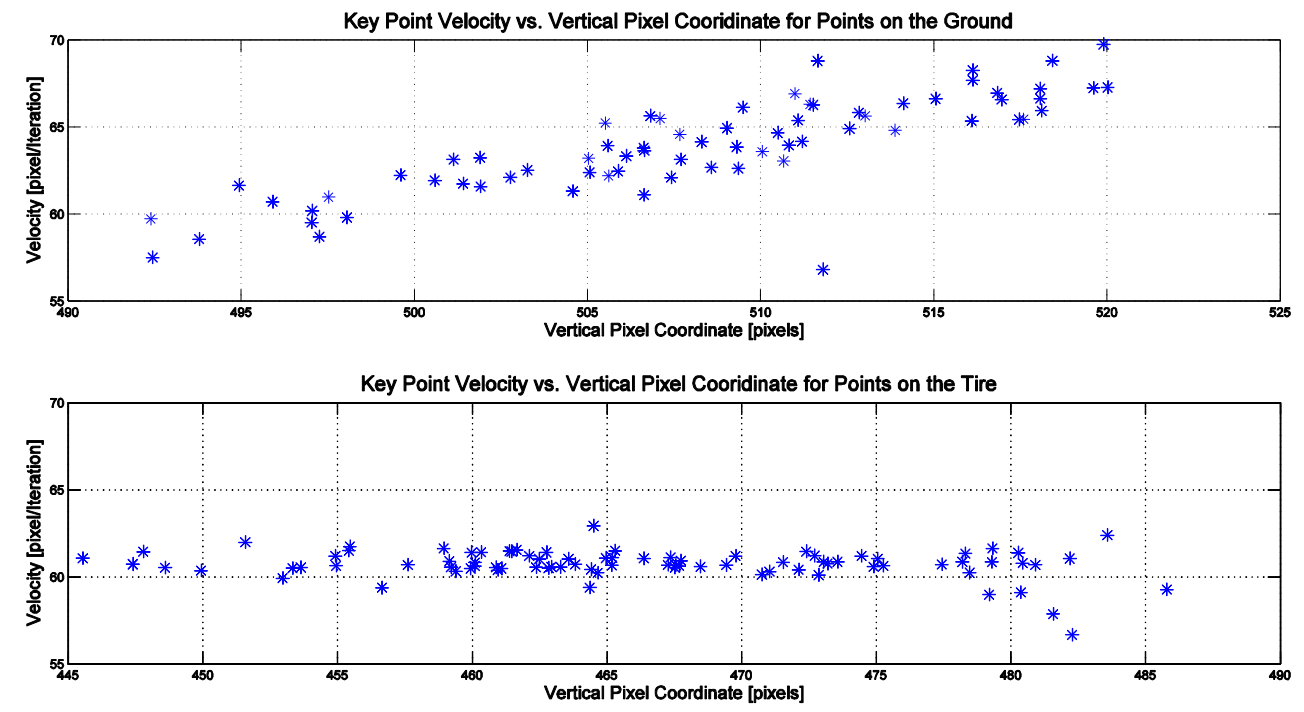

Figure 5: Velocity of key points versus vertical pixel coordinate for points on the road (top) and points on the tyre (bottom)

$\left(V_{i}\right)$ to be much larger than the velocity measured at $K P_{j}\left(V_{j}\right)$. To compensate for this effect all velocities must be transferred to the velocity measured at the contact point. The change in depth for the road can be captured by the vertical pixel coordinate, Figure 5 shows a plot of the key point velocity against the vertical pixel coordinate of the key point. From this figure it is evident that there is a substantial change in velocity with respect to a change in vertical pixel location for the key points located on the road. Comparatively there is no noticeable change in velocity for key points located on the tyre. As such the tyre velocity is simply taken as the average of all key points on the tyre. However, for the road the parallax effect must be compensated for. It is also noticeable from the figure that the relationship between the vertical pixel coordinate and the velocity of the road key points seems to be linear in nature. Thus, a straight line is fitted through these points to obtain a model for this relationship. This is achieved by making use of the RANSAC algorithm. However, in this RANSAC algorithm all the inlier points for the best model are used to fit a linear Least Means Square fit between all the inlier points. This effectively bases the fit on inlier points only, removing any outliers which could distort the relationship. This process is performed at each iteration since the model changes as a result of a change in the vertical displacement of the tyre i.e. the bouncing of the tyre. Additionally the fit from the previous iteration is used as the first model in the current RANSAC algorithm. Once this model has been obtained the model is simply used to determine the velocity at the contact point $R_{y, t+1}$. 
It is further assumed that the velocity of the camera relative to the tyre and ground is minimal in comparison to the velocity of the tyre and the ground. The camera motion therefore would not affect the measurement of the tyre and ground velocities. In the tests conducted no discernible camera motion could be observed which would affect the velocity measurements. If the vehicle motion becomes slow then a variable frequency sampling method can be used to measure the velocities over more than one frame therefore increasing the observed motion and reducing the camera motion.

From this process the velocity of the tyre and road has been determined at the contact point from which the slip ratio is determined from:

$$
s=-\frac{V_{\text {road }}-V_{\text {tyre }}}{V_{\text {road }}}
$$

\section{EXPERMENTAL SETUP AND RESULTS}

The method is validated by comparing it to the traditional method of estimating the slip-ratio from eq. (1). The method is tested on a tyre test rig as shown in Figure 5. The tyre test rig is equipped with a wheel force transducer, developed by the University of Pretoria, which can measure all six force/moment components as well as the rotational angle of the wheel. A road profiling laser is used to measure the effective tyre rolling radius and a differential GPS is used to measure the test rig speed.

The tyre test rig is pulled using a large truck with the vehicle speed kept as constant as possible. The test rig is equipped with an electronically controlled pneumatic braking system which is programmed to provide a gradual increase in braking, thus generating a gradually higher slip-ratio. The braking is performed at the input of an open differential which is attached to both wheels instead of at the wheels directly. The differential unfortunately has the effect that the wheel rotation can become negative close to lockup. A Point Grey Flea3 (USB 3.0) camera is mounted facing the contact patch of the tyre, as shown encircled in Figure 6. The camera is sampled and synchronized with the data by using a $100 \mathrm{~Hz}$ trigger. During the braking test a fairly low resolution grey-scale video of 640x480 pixels is captured of the contact patch region. The slip-ratio is then determined in post processing from the recorded video. 


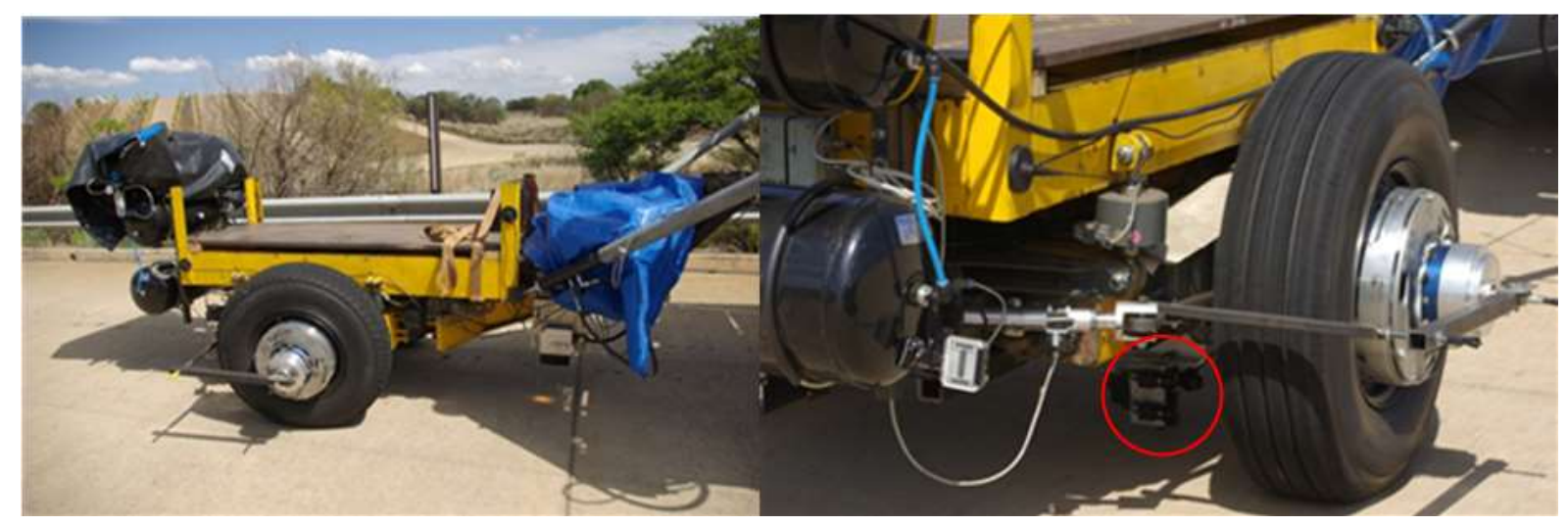

Figure 6: Tyre test rig, showing encircled camera mounting position
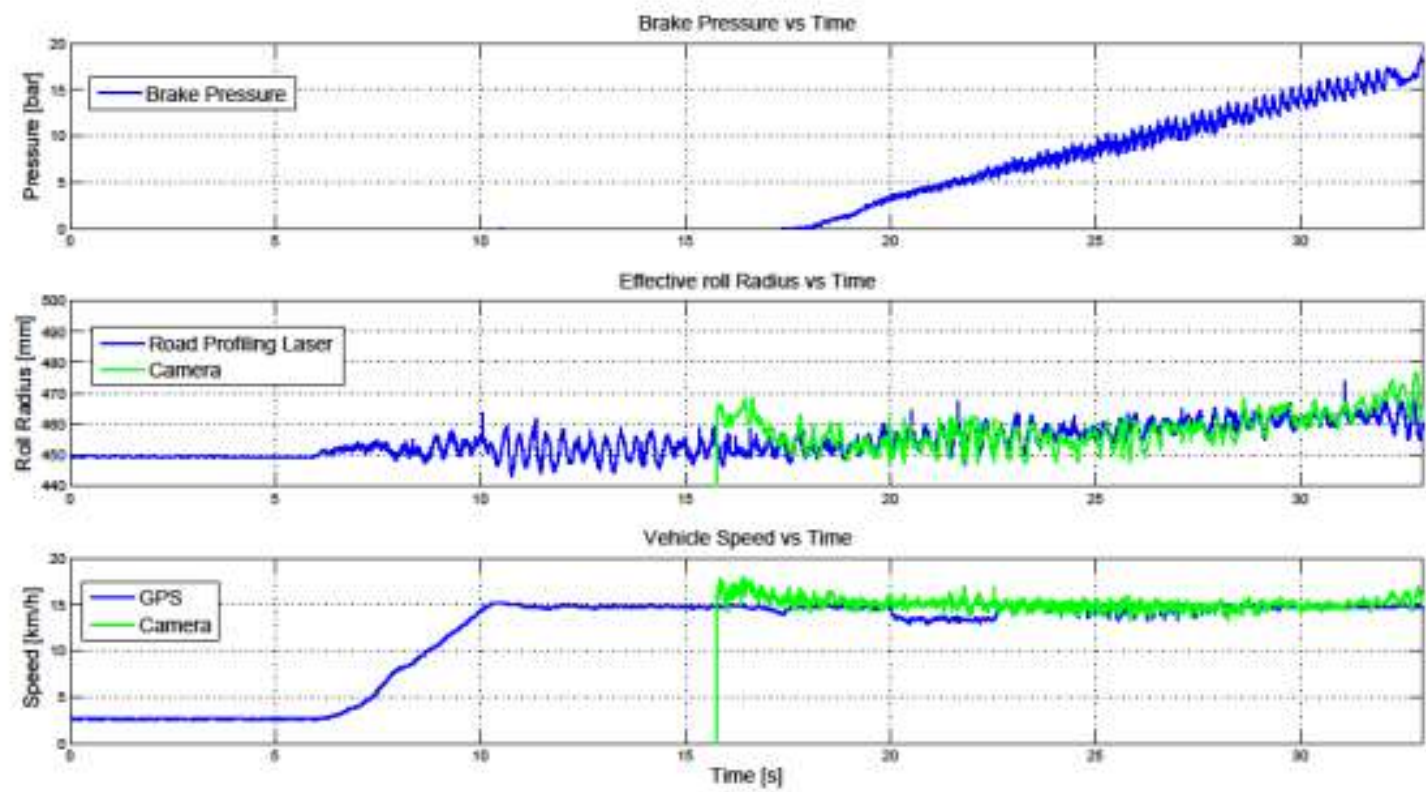

Figure 7: Hydraulic brake pressure, comparison between effective roll radius of a road profiling laser and the camera system, vehicle speed comparison between GPS and camera system.

In order to compare the measurements obtained using the camera, with units in pixels, to the measurement obtained with traditional means required the scaling of the camera measurements. The comparison of effective roll radius and vehicle speed along with the hydraulic brake pressure is presented in Figure 7 . The data obtained from the camera starts from $16 \mathrm{~s}$ and synchronised using the trigger signal. The effective roll radius plot, in the middle, illustrates the change in roll radius as a result of the load transferred, depicted by the gradual increase, as well as the bouncing of the tyre test rig depicted by the oscillatory nature of the graph. The plot also shows that initially the camera roll radius estimation has a slight error, however, as the test continues better correlation is obtained with the camera capturing both the gradual increase as well as oscillatory change in the tyre effective roll radius. The last plot depicts the vehicle speed obtained from the GPS as well as the camera. It can be 
observed that the camera shows good correlation overall but contains a gradual decrease and is more oscillatory. These effects are caused by the change in camera position relative to the road, and can be noted to be the inverse of the effective roll radius measurement from the camera. This is because the change in camera position creates a slight change in parallax, however this phenomena occurs on the tyre velocity measurement as well, depicted in Figure 8, effectively negating this effect. It should also be stated that the dip in GPS obtained vehicle velocity between 20 s and $30 \mathrm{~s}$ is due to a loss of a satellite and is therefore not a true representation of the actual vehicle speed.
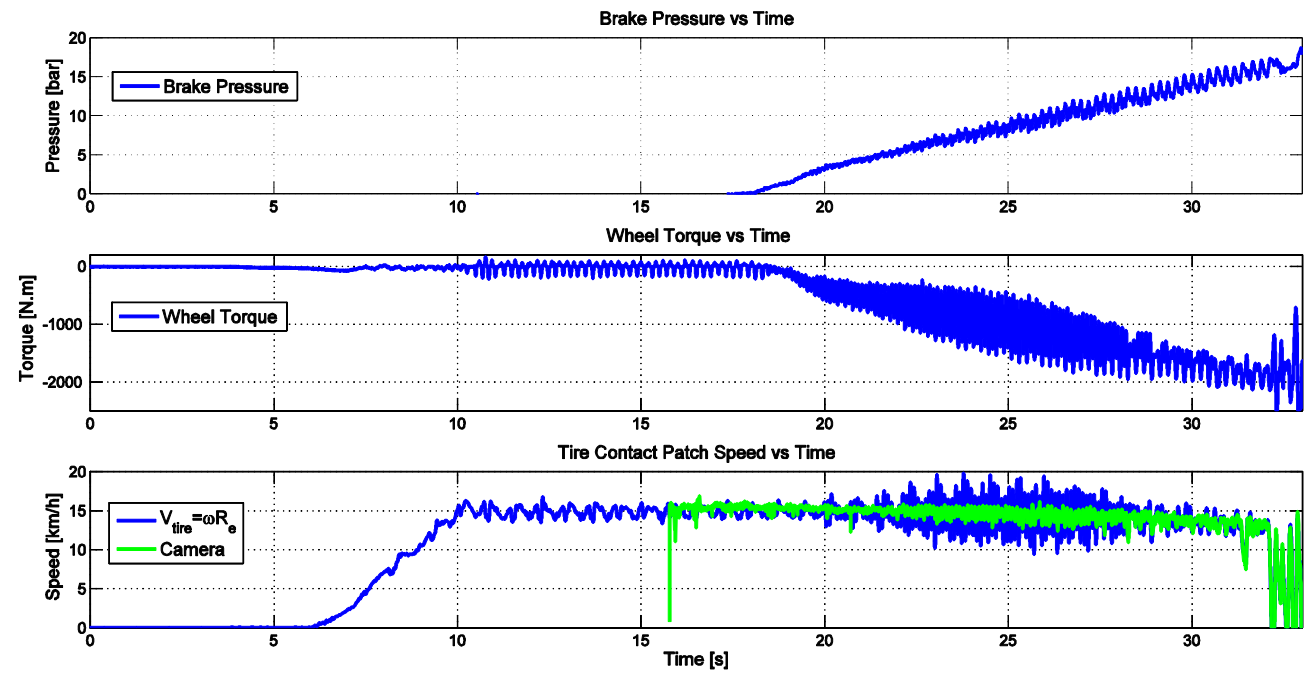

Figure 8: Hydraulic brake pressure, wheel torque and estimated tyre contact patch speed comparison between the camera based method and using the effective roll radius and wheel rotation speed.

Figure 8 also shows the wheel torque measured at the wheel (middle plot). It is evident from this plot that the torque has a high degree of oscillation. The same oscillations are evident from the brake pressure. These oscillations were caused by misalignment in the brake system. These oscillations also caused the arm which creates a fixed reference for the angle sensor in the Wheel Force Transducer to vibrate at the same frequency. As a result of this an artificial signal is generated on angle measurement, which after differentiating to obtain the wheel rotational speed is amplified. The result is that the slip measurement during the large oscillations is largely erroneous. This is better illustrated in Figure 9 showing an enlarged region containing the oscillations, and the region with less oscillatory behaviour. While it is evident that the oscillatory brake torque should result in an oscillatory tyre speed, the excessive oscillations from the wheel rotational speed sensor is largely noise. This noise generated by the fixed reference moving is a problem associated with this technique to measure wheel speed. The fixed reference will in general not be a rigid connection with not only the arm being flexible 

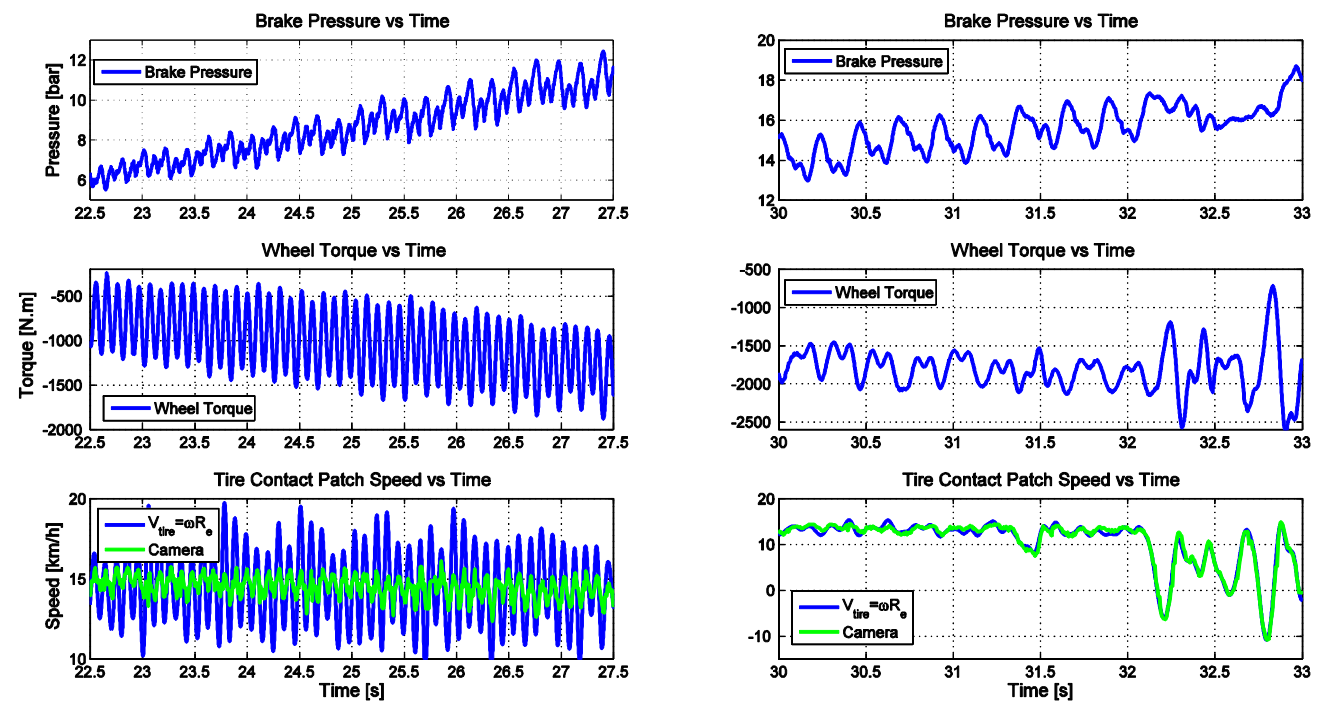

Figure 9: Enlarged regions of the hydraulic brake pressure, wheel torque and estimated tyre contact patch speed comparison between camera based method and using the effective roll radius and wheel rotation speed.

but the mounting point may have experience motion which could induce noise on the measured angle. A better alternative is to use a proximity based measurement often used in ABS systems to measure wheel speed. However, even these measurements can be corrupted with noise due to manufacturing tolerances, corrosion, dirt, low-frequency disturbances due to varying air gap between sensor head and the sensor wheel and disturbances on the transmission path [17].

The camera based tyre speed shows the same trend however at a reduced magnitude. In the regions where the oscillatory wheel torque is much less pronounced, which also resulted in less induced noise on the angle sensor, shows good correlation between the contact patch tyre speed obtained from the rotational wheel speed sensor and the camera based measurement.

Figure 10 depicts the comparison of the slip-ratio determined from the camera system to that of the traditional method using eq. (1). Again the oscillatory nature of the method from eq. (1) is evident from the top plot, if the region from 30-33 seconds is compared, as shown in the bottom plot, excellent comparison of the slip-ratio is shown. It should be noted that the slip-ratio values exceeds $100 \%$, this is due to the tyre velocity actually becoming negative. The regions with values over $100 \%$ should be inverted to be negative, however the graph is better displayed by keeping these values positive. It is also noted that there is a slight phase lag between the signals of $10 \mathrm{~ms}$, this value however falls within the sampling frequency of the camera. It is therefore impossible to say with the current data whether the slip-ratio generated at the tyre is indeed out of 

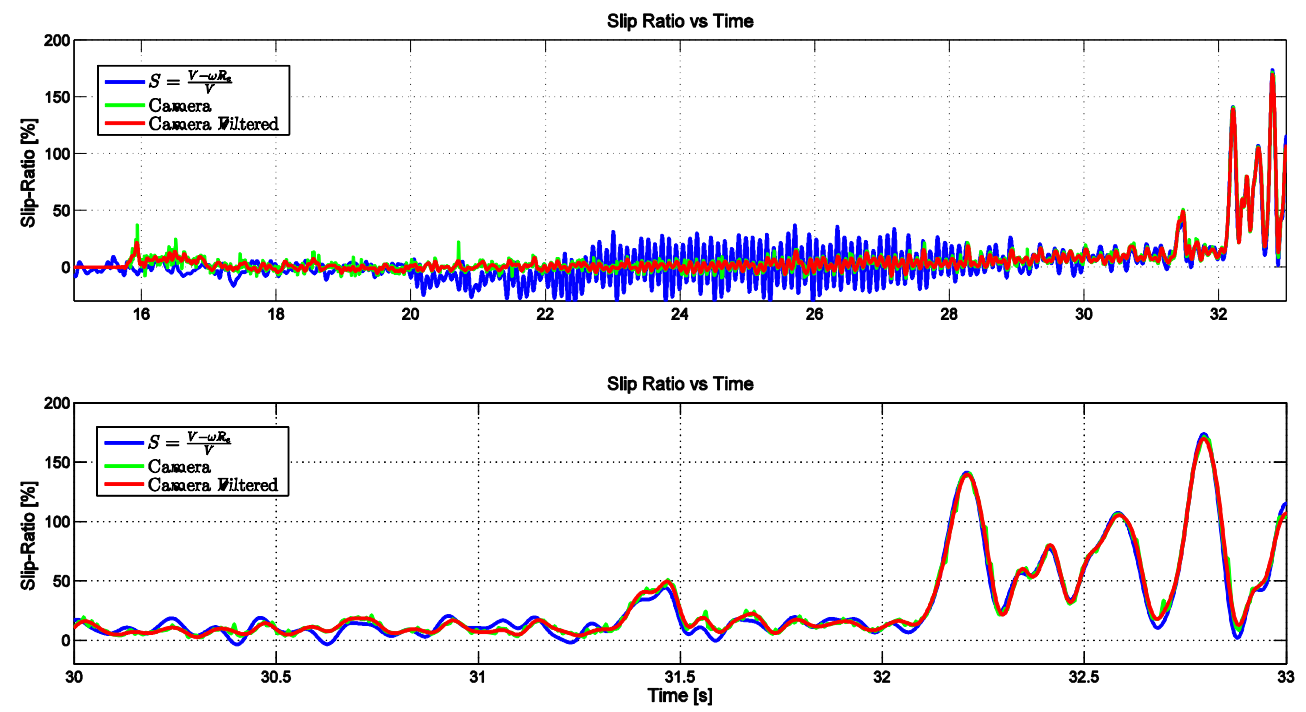

Figure 10: Slip-ratio comparison between the camera based method and the traditional method using (1)

phase of that determined using the rim rotational speed. For an analysis into the phase difference a higher frequency is required, as well as, good control over the phase shifts caused by any filtering process during sampling. The longitudinal force as a function of tyre longitudinal slip-ratio is displayed in Figure 11. This figure clearly illustrates that the relationship obtained from the camera presents typical results expected from such a test [10] with the maximum force being produced around 15\% slip. It can be concluded that the methodology represents an inexpensive alternative method to measuring the slip-ratio of a tyre.

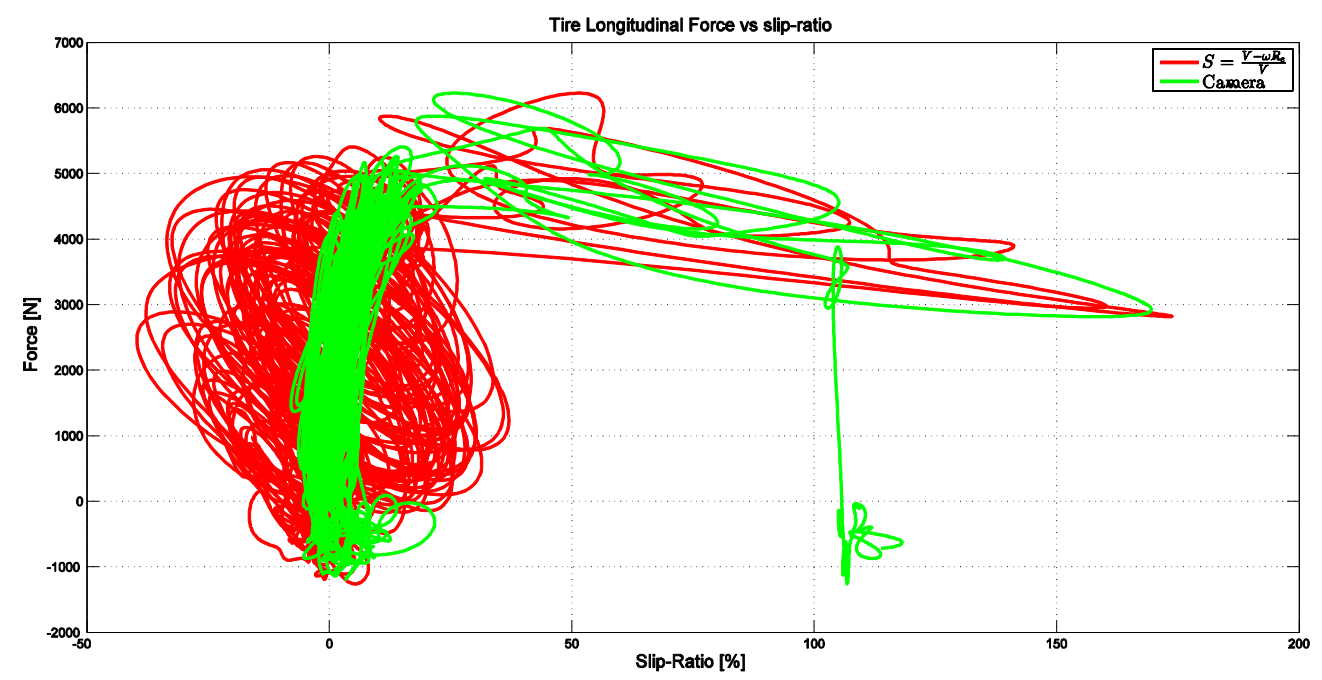

Figure 11: Longitudinal tyre force vs. slip-ratio comparison using slip-ratio based from (1) and the measured using a camera. 
Measuring of the Rolling radius, tyre and road velocities has been shown to be possible on smooth land terrain. The following challenge is to test the system on rough terrain. The challenges on rough road are more complex since the tyre deformation is more complex. The tyre contact patch may have various rolling radius depending on the tyre deformation as well as wheel camber. This can partly be solved by using cameras on either side of the tyre or even by placing a camera inside of the tyre. This would provide more and better information than the traditional method used to measure the slip ratio. Additionally radial lines can be made on the tyre to observe the deformation of the tyre while rolling. This method could therefore be used to aid in the understanding the generation of slip at the contact patch.

The effect of dust on the measurement of the slip-ratio when measuring over deformable terrain such as very sand should also be investigated. If the amount of dust is so severe that the tyre and terrain is not visible at all then the method would fail to measure the slip-ratio. In conditions where the view is partially blocked by dust the system should work provided that features on the tyre and road can be identified in both views. The amount of features tracked may be reduced, which could affect accuracy, but only a few points are needed for the system to measure the slip-ratio.

\section{CONCLUSION}

In this paper a method whereby the longitudinal slip ratio of a tyre can be measured is presented. The method consists of an inexpensive camera mounted such that it captures images of the tyre -road interface. Digital Image Correlation (DIC) techniques are used to track points on both the tyre and road at the contact interface. A clustering methodology is used to differentiate between points on the tyre and point on the road, as well as, remove outliers. The method also tracks the vertical height of the contact patch, thus effectively measuring the effective roll radius. This allows for the method to compensate for parallax errors which are

introduced as the tyre deflection changes. The method is shown to accurately estimate the slip-ratio when compared to traditional methods of estimating the slip-ratio of a tyre. 
NOTATION

\begin{tabular}{|c|c|}
\hline $\boldsymbol{H}$ & Homography matrtix \\
\hline $\boldsymbol{h}_{\boldsymbol{i}}$ & $\mathrm{i}^{\text {th }}$ Row of homography matrix \\
\hline$I_{t}$ & Frame at time $t$ \\
\hline$K P_{j}$ & Key point $j$ \\
\hline$q$ & Homogeneous coordinate of point in image $I_{t}$ \\
\hline$q^{\prime}$ & Homogeneous coordinate of point in image $I_{t+1}$ \\
\hline$R_{e}$ & Effective rolling radius \\
\hline$R_{y, t}$ & Estimated vertical contact point in image at time $t$ \\
\hline$S$ & Tyre Longitudinal slip ratio \\
\hline$V$ & Longitudinal vehicle speed \\
\hline$V_{\text {road }}$ & Image velocity of road \\
\hline$V_{t}$ & Theoretical velocity of tyre \\
\hline$V_{\text {tyre }}$ & Image velocity of tyre at contact patch \\
\hline$V_{x}$ & Actual tyre velocity \\
\hline$X_{i k}$ & Cluster parameter $k$ of cluster $i$ \\
\hline$\omega$ & Wheel rotational speed \\
\hline
\end{tabular}

\section{ACKNOWLEDGEMENTS}

The financial assistance of the National Research Foundation (DAAD-NRF) towards this research is hereby acknowledged. Opinions expressed and conclusions arrived at, are those of the author and are not necessarily to be attributed to the DAAD-NRF.

\section{REFERENCES}

[1] Deur J, Ivanović V, Troulis M, Miano C, Hrovat D, Asgari J. (2005) Extensions of the LuGre tyre friction model related to variable slip speed along the contact patch length, Vehicle System Dynamics: International Journal of Vehicle Mechanics and Mobility. 2005;43:sup1, pp. 508-524.

[2] Sakai H. Theoretical and Experimental Studies on the Dynamic Properties of Tyres Part 1: Review of theories of rubber friction. International Journal of Vehicle Design. 1981;2, pp. 78-110 
[3] Wong, J.Y. Theory of Ground Vehicles, Third Edition, John Wiley \& Sons. 2001.

[4] Hüsemann T. Wöhrmann M. The Impact of Tire Measurement Data on Tire Modeling and Vehicle Dynamics Analysis. Tire Science and Technology, TSTCA, 2010;38:2, pp. 155-180.

[5] Cabrera JA, Ortiz A, Simon A, Garcia G, La Blanca P. A Versatile Flat Track Tire Testing Machine. Vehicle System Dynamics: International Journal of Vehicle Mechanics and Mobility 2003;40:4, pp. 271-284.

[6] Miller SL, Youngberg B, Millie A, Schweizer P. Gerdes JC. Calculating Wheel Slip and Tire Pareameters Using GPS Velocity. Proceedings of the Amercian Control Conference, 2001, p. 1800 -1805. Arlington, VA June 25-27

[7] Rill G. First Order Tire Dynamics, II European Conference on Computational Mechanics Solids, Structures and Coupled Problems in Engineering C.A. Mota Soares et.al. (eds.) 2006. Lisbon, Portugal, 5-8 June

[8] Clover CL, Bernard JE. Longitudinal Tire Dynamics. Vehicle System Dynamics: International Journal of Vehicle Mechanics and Mobility, 1998;29:4, pp. 231-260

[9] Mavros G, Rahnejat H, King PD. Transient analysis of tyre friction generation using a brush model with interconnected viscoelastic bristles. Proceeding of the Institution of Mechanical Engineers, Journal Multi-Body Dynamics. 2004;219

[10] Zanten A, Erhardt R, Lutz A. Measurement and Simulation of Transients in Longitudinal and Lateral Tire Forces. SAE Technical Paper 900210. 1990

[11] Xavier J, Sousa AMR, Morais, JJL, Filipe, VMJ, Vaz M. Measuring displacement fields by cross-correlation and a differential technique: experimental validation. Optical Engineering. 2012;51:4

[12] Lowe D. Distinctive image features from scale-invariant keypoints. International Journal of Computer Vision. 2004;60:2, pp. 91-110

[13] Bay H, Ess A, Tuytelaars T, Van Gool L.SURF: Speeded Up Robust Features. Computer Vision and Image Understanding (CVIU), 2008;110:3, pp. 346 - 359

[14] Gupta A, Gupta A, Mishra A. Research Paper on Cluster Techniques of Data Variations. International Journal of Advance Technology \& Engineering Research (IJATER) 2011;1: 1

[15] Hartley R, Zisserman A. Multiple View Geometry in Computer Vision. Cambridge University Press. 2000

[16] Fischler MA, Bolles RC. Random sample consensus: A paradigm for model fitting with applications to image analysis and automated cartography. Communications of the ACM, 1981;4:6, pp. 381-395.

[17] Schwarz R, Nelles O, Scheerer P, Isermann R. Increasing Signal Accuracy of Automotive Wheel-Speed Sensors by Online Learning. Proceedings of the American Control Conference, 1997. Albuquerque, New Mexico. 